\title{
Grußwort zum 75. Geburtstag von Herrn Prof. Dr. E. G. Jung
}

Greeting on the Occasion of the 75th Birthday of Prof. Dr. E. G. Jung

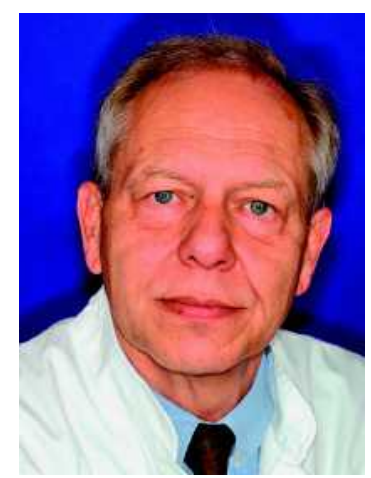

Prof. Dr. Harald Gollnick

\author{
Bibliografie \\ DOI $10.1055 / \mathrm{s}-2007-966298$ \\ Akt Dermatol 2007; 33: 64 \\ (c) Georg Thieme Verlag KG . \\ Stuttgart · New York \\ ISSN 0340-2541
}

Korrespondenzadresse

Prof. Dr. med. Harald Gollnick

Universitätsklinik für

Dermatologie und Venerologie

Med. Fakultät der Otto-von-

Guericke-Universität Magdeburg

Leipziger Str. 44

39120 Magdeburg

harald.gollnick@medizin.uni-

magdeburg.de
Lieber Jubilar, lieber Herr Kollege Jung,

es ist mir eine ganz besondere Freude, Ihnen als Präsident im Namen des Vorstandes der Deutschen Dermatologischen Gesellschaft zum 75. Geburtstag sehr herzlich zu gratulieren.

Sie haben sich in vorbildlicher Weise in Ihrer akademischen Laufbahn, aber auch nach der Zeit Ihrer Emeritierung für unser Fachgebiet Dermatologie und Venerologie stets und erfolgreich eingesetzt. In den Jahrzehnten Ihres Wirkens haben Sie entscheidende Beiträge auf dem Gebiet der Photobiologie unter anderem mit der Forschergruppe an der Heidelberger Universitätshautklinik zur Thematik „Biologie und Pathologie der Lichtwirkung" und kontinuierlich in Ihrer Mannheimer Zeit geleistet. Ganz im Vordergrund stand Ihre Forschung zur Pathogenese des Xeroderma pigmentosum. Sie haben wesentlich und kontinuierlich zur Entwicklung der modernen Phototherapie, der Prävention gegen UV-Strahlen, zur Aufklärung von photoallergischen Reaktionen und von seltenen, genetisch bedingten Dermatosen in Klinik und Forschung beigetragen. Einer von vielen Höhepunkten Ihrer wissenschaftlichen Laufbahn war sicherlich auch die Präsidentschaft in der European Society of Dermatological Research gewesen.

Nicht nur die Zeitschrift „Photodermatology“ haben Sie mitgeprägt, die Zeitschrift „Aktuelle Dermatologie" ist ohne Ihre Herausgeberschaften gar nicht denkbar, sie ist Ihre Zeitschrift und trägt Ihre Handschrift. Nicht zu vergessen Ihr „Lehrbuch der Dermatologie“, das seinerzeit 1989 einen neuen Stil der Lehrstoffpräsentation aufzeigte und heute in der 6 . Auflage von Ihrer Schülerin Prof. Moll weitergeführt wird.
Über viele Jahre haben Sie sich auch innerhalb der DDG als Vorstandsmitglied und im Rahmen der Melanomkommission für unser Fachgebiet in Hinsicht auf dieses bedeutende Tumorleiden klinisch und wissenschaftlich eingesetzt.

Sie sind auch einer der Dermatologen, die durch Ihr wissenschaftliches Arbeiten und den Blick über die Fachgrenzen national und international hinaus weltweite Anerkennung gefunden haben. Dies hat man auch in Ihrer Fakultät erkannt, die Sie über drei Jahre als Dekan geleitet haben. Schließlich wurden Sie auch zum Prorektor der Universität Heidelberg von 1995 bis 1997 gewählt, ein Amt, das nur wenige Dermatologen als Auszeichnung ihrer akademischen Laufbahn erreichen können. Mit Weitsicht haben Sie die Mannheimer Hautklinik modernisiert und den Grundstock dafür gelegt, dass Ihr Nachfolger Prof. Goerdt nun in diesem Jahr den Neubau der Mannheimer Hautklinik wird beziehen können. In Ihrer langen akademischen Laufbahn als Kliniker und akademischer Lehrer haben Sie viele Assistentinnen und Assistenten ausgebildet, die heute mit Stolz auf ihren Lehrer schauen und Ihnen sicherlich und zurecht größte Dankbarkeit entgegenbringen.

Die Deutsche Dermatologie ist stolz, hervorragende Leistungsträger wie Sie in Ihren Reihen zu haben, die unserem Fach gutgetan haben und sich auch weiterhin für uns einsetzen. Hierfür danken wir Ihnen!

\section{Alles Gute in Ihrem aktiven Ruhestand!}

Prof. Dr. H. Gollnick

Präsident der Deutschen Dermatologischen Gesellschaft 